PROCEEDINGS OF THE

AMERICAN MATHEMATICAL SOCIETY

Volume 132, Number 4, Pages 1143-1151

S 0002-9939(03)07257-5

Article electronically published on August 28, 2003

\title{
AN ESTIMATE FOR THE NUMBER OF BOUND STATES OF THE SCHRÖDINGER OPERATOR IN TWO DIMENSIONS
}

\author{
MIHAI STOICIU
}

(Communicated by Joseph A. Ball)

\begin{abstract}
For the Schrödinger operator $-\Delta+V$ on $\mathbb{R}^{2}$ let $N(V)$ be the number of bound states. One obtains the following estimate:

$$
N(V) \leq 1+\int_{\mathbb{R}^{2}} \int_{\mathbb{R}^{2}}|V(x)||V(y)|\left|C_{1} \ln \right| x-y\left|+C_{2}\right|^{2} d x d y
$$

where $C_{1}=-\frac{1}{2 \pi}$ and $C_{2}=\frac{\ln 2-\gamma}{2 \pi}$ ( $\gamma$ is the Euler constant). This estimate holds for all potentials for which the previous integral is finite.
\end{abstract}

\section{INTRODUCTION}

On $\mathbb{R}^{3}$, there is a well-known bound for the number of bound states $N(V)$ discovered by Birman 3 and Schwinger [9]:

$$
N(V) \leq \frac{1}{(4 \pi)^{2}} \int_{\mathbb{R}^{3}} \int_{\mathbb{R}^{3}} \frac{|V(x)||V(y)|}{|x-y|^{2}} d x d y .
$$

The method of proof is the "Birman-Schwinger principle", which states that for a potential $V \leq 0$ and for a number $E<0$ :

$$
N_{(-\infty, E]}(-\Delta+V)=N_{[1, \infty)}\left(|V|^{1 / 2}(-\Delta-E)^{-1}|V|^{1 / 2}\right)
$$

where $N_{I}(T)$ denotes the number of eigenvalues (counting multiplicities) of the operator $T$ in the interval $I$.

The operator $(-\Delta-E)^{-1}$ has integral kernel

$$
G_{3}(x, y, E)=\frac{1}{4 \pi}|x-y|^{-1} e^{-\sqrt{-E}|x-y|},
$$

which converges when $E \uparrow 0$ (for $x \neq y$ ). This implies that an estimate for $N(V)$ can be obtained by estimating $N_{(-\infty, E]}(-\Delta+V)$ first and then taking $E \uparrow 0$. A detailed proof of this result can be found in [12].

This proof does not work in two dimensions since the integral kernel of $(-\Delta+$ $V)^{-1}$ contains $\ln (\sqrt{-E}|x-y|)$ which diverges as $E \uparrow 0$.

Received by the editors December 17, 2002.

2000 Mathematics Subject Classification. Primary 35P15, 35J10; Secondary 81Q10.

(C)2003 American Mathematical Society 
Khuri, Martin and Wu conjectured in [5] the following bound for $N(V)$ in two dimensions:

$$
\begin{aligned}
N(V) \leq 1 & +C_{1} \int_{\mathbb{R}^{2}}|V|^{*}(x)\left(\ln \left|\frac{x_{0}}{x}\right|\right)^{+} d x \\
& +C_{2} \int_{\mathbb{R}^{2}}\left|V_{-}(x)\right|\left(\ln \left|\frac{x}{x_{0}}\right|\right)^{+} d x+C_{3} \int_{\mathbb{R}^{2}}\left|V_{-}(x)\right| d x
\end{aligned}
$$

where $x_{0} \neq 0$ and $|V|^{*}$ denotes the symmetric decreasing rearrangement of $|V|$.

Our goal is to find bounds similar to (1.1) using the "Birman-Schwinger principle" and a method discovered by Simon in [11. The idea is to write the integral kernel of $|V|^{1 / 2}(-\Delta-E)^{-1}|V|^{1 / 2}$ as the sum of a Hilbert-Schmidt operator and a rank-one perturbation and then apply the Birman-Schwinger method.

The main results in this paper are Theorem 3.3 and Proposition 3.4 which give two bounds for $N(V)$ in two dimensions:

$$
B(V)=1+\int_{\mathbb{R}^{2}} \int_{\mathbb{R}^{2}}|V(x)||V(y)|\left|-\frac{1}{2 \pi} \ln \right| x-y\left|+\frac{\ln 2-\gamma}{2 \pi}\right|^{2} d x d y
$$

and

$$
\begin{aligned}
\widetilde{B}(V)=1 & +C_{3}\|V\|_{1}^{2}+C_{4}\|V\|_{1} \int_{\mathbb{R}^{2}}|V(x)|[\ln (1+|x|)]^{2} d x \\
& +C_{5}\|V\|_{1} \int_{\mathbb{R}^{2}}|V|^{*}(x)[\ln |x|]^{2} \chi_{\{|x| \leq 1\}} d x
\end{aligned}
$$

These bounds are similar to the one conjectured in [5] with the difference that for large coupling constants, $B(\lambda V), \widetilde{B}(\lambda V) \sim \lambda^{2}$ whereas the bound conjectured by Khuri, Martin and $\mathrm{Wu}$ is $\sim \lambda$.

\section{Estimate for nice potentials}

One can prove the following:

Theorem 2.1. Let $V \in L^{\infty}\left(\mathbb{R}^{2}\right)$ be a real-valued compactly supported potential. Then

$$
N(V) \leq 1+\int_{\mathbb{R}^{2}} \int_{\mathbb{R}^{2}}|V(x)||V(y)|\left|C_{1} \ln \right| x-y\left|+C_{2}\right|^{2} d x d y
$$

where $C_{1}=-\frac{1}{2 \pi}$ and $C_{2}=\frac{\ln 2-\gamma}{2 \pi}(\gamma \approx 0.577$ is the Euler constant $)$.

Proof. Since $V \in L^{\infty}\left(\mathbb{R}^{2}\right)$ and $\operatorname{supp}(V)$ is compact, it follows that $V \in L^{2}\left(\mathbb{R}^{2}\right)$. So $(-\Delta+V)$ is a well-defined selfadjoint operator with $\sigma_{\text {ess }}(-\Delta+V)=[0, \infty)$.

Without loss of generality, one can assume $V \leq 0$. Indeed, if $V_{-}=\max (-V, 0)$ is the negative part of $V$, then

$$
-\Delta+\left(-V_{-}\right) \leq-\Delta+V .
$$

So by the min-max principle, $N(V) \leq N\left(-V_{-}\right)$.

Now, from the Birman-Schwinger principle one gets, for $E<0$ :

$$
N_{(-\infty, E]}(-\Delta+V)=N_{[1, \infty)}\left(|V|^{1 / 2}(-\Delta-E)^{-1}|V|^{1 / 2}\right) .
$$

The integral kernel of $(-\Delta+E)^{-1}$ is (cf. [1] and [2]):

$$
G_{2}(x, y, E)=\frac{1}{2 \pi} K_{0}(\sqrt{-E}|x-y|)
$$


where $K_{0}$ is a modified Bessel function. In particular,

$$
K_{0}(x)=-(\ln x) I_{0}(x)+h(x)
$$

where the Bessel function $I_{0}$ and the function $h$ (defined on $\mathbb{R}$ ) are real-valued analytic functions with $I_{0}(0)=1$ and $h(0)=\ln 2-\gamma$. Let $\varphi \in C_{0}^{\infty}\left(\mathbb{R}^{2}\right)$ be such that $\varphi(x)=1$ for $|x| \leq 1$ and $\varphi(x)=0$ for $|x| \geq 2$. Then, for $f(x)=-\frac{1}{2 \pi} I_{0}(x) \varphi(x)$ and $g(x)=\frac{1}{2 \pi} K_{0}(x)-f(x) \ln (x)$, one gets

$$
\frac{1}{2 \pi} K_{0}(x)=(\ln x) f(x)+g(x)
$$

The functions $f$ and $g$ are in $C^{\infty}(\mathbb{R})$, with $f(0)=-\frac{1}{2 \pi}$ and $g(0)=\frac{\ln 2-\gamma}{2 \pi}$. Furthermore, $f$ has compact support and $g$ has exponential decay at infinity (since the modified Bessel function $K_{0}$ has exponential decay at infinity). Using (2.1) and (2.3) one gets

$$
\begin{aligned}
G_{2}(x, y, E)= & {[\ln (\sqrt{-E}|x-y|)] f(\sqrt{-E}|x-y|)+g(\sqrt{-E}|x-y|) } \\
=\ln \sqrt{-E}\left[f(\sqrt{-E}|x-y|)+\frac{1}{2 \pi}\right]+\ln |x-y| f(\sqrt{-E}|x-y|) & \\
& \quad+g(\sqrt{-E}|x-y|)+\left[-\frac{1}{2 \pi} \ln (\sqrt{-E})\right] .
\end{aligned}
$$

One can write $\left(|V|^{1 / 2}(-\Delta-E)^{-1}|V|^{1 / 2}\right)=A_{E}+B_{E}$ where $A_{E}$ and $B_{E}$ are operators on $L^{2}\left(\mathbb{R}^{2}\right)$ defined by

$$
\begin{gathered}
A_{E} \varphi(x)=\int_{\mathbb{R}^{2}}|V(x)|^{1 / 2}\left[\ln \sqrt{-E}\left(f(\sqrt{-E}|x-y|)+\frac{1}{2 \pi}\right)\right. \\
+\ln |x-y| f(\sqrt{-E}|x-y|)+g(\sqrt{-E}|x-y|)]|V(y)|^{1 / 2} \varphi(y) d y, \\
B_{E} \varphi(x)=|V(x)|^{1 / 2} \int_{\mathbb{R}^{2}}\left[-\frac{1}{2 \pi} \ln (\sqrt{-E})|V(y)|^{1 / 2}\right] \varphi(y) d y .
\end{gathered}
$$

$B_{E}$ is therefore a selfadjoint rank-one operator with range $\mathbb{C}|V|^{1 / 2}$.

Now, in order to estimate the number of eigenvalues greater than or equal to 1 of the operator $A_{E}$, the following lemma will be useful.

Lemma 2.2. Let $V, E, f, g, A_{E}$ be as before, and let $F_{E}$ be the integral kernel of $A_{E}$. Then $F_{E} \in L^{2}\left(\mathbb{R}^{2} \times \mathbb{R}^{2}\right)$.

Proof. The potential $V$ is compactly supported. So let $R>0$ be such that $\operatorname{supp}(V) \subset\left\{x \in \mathbb{R}^{2},|x| \leq R\right\}$.

Since $f$ and $g$ are bounded, one immediately gets that, for any $E<0$, the functions $\ln (\sqrt{-E})\left(f(\sqrt{-E}|x-y|)+\frac{1}{2 \pi}\right), f(\sqrt{-E}|x-y|)$ and $g(\sqrt{-E}|x-y|)$ are bounded on $\mathbb{R}^{2}$.

A simple computation shows that

$$
\int_{|x| \leq R} \int_{|y| \leq R}(\ln |x-y|)^{2} d x d y \leq \pi R^{2} \int_{|z| \leq 2 R}(\ln |z|)^{2} d z<\infty
$$

and therefore, since $V \in L^{\infty}\left(\mathbb{R}^{2}\right)$, one can conclude that $F_{E} \in L^{2}\left(\mathbb{R}^{2} \times \mathbb{R}^{2}\right)$.

From the previous lemma, it follows immediately that $A_{E}$ is a selfadjoint HilbertSchmidt operator. Therefore, one can estimate $N_{[1, \infty)}\left(A_{E}\right)$ as in the proof of the 
Birman-Schwinger theorem and, denoting by $S$ the set of eigenvalues of $A_{E}$, one gets

$$
\begin{aligned}
N_{[1, \infty)}\left(A_{E}\right) & \leq \sum_{\lambda \in S}|\lambda|^{2}=\left\|A_{E}\right\|_{2}^{2}=\left\|F_{E}\right\|_{2}^{2} \\
& \left.=\int_{\mathbb{R}^{2}} \int_{\mathbb{R}^{2}} \mid F_{E}(x, y)\right)\left.\right|^{2} d x d y .
\end{aligned}
$$

( $\left\|A_{E}\right\|_{2}$ denotes the Hilbert-Schmidt norm of the operator $A_{E}$.) Since $B_{E}$ is a rank-one operator, the eigenvalues of $A_{E}$ and $\left(A_{E}+B_{E}\right)$ interlace. So

$$
\begin{aligned}
N_{(-\infty, E]}(-\Delta+V) & =N_{[1, \infty)}\left(A_{E}+B_{E}\right) \\
& \left.\leq 1+\int_{\mathbb{R}^{2}} \int_{\mathbb{R}^{2}} \mid F_{E}(x, y)\right)\left.\right|^{2} d x d y .
\end{aligned}
$$

Now, in order to obtain an estimate for $N(V)$ one has to take $E \uparrow 0$. The following lemma shows that the previous integral converges as $E$ approaches 0 .

Lemma 2.3. Let $V, E$ and $F_{E}$ be as before. Then

$$
\begin{aligned}
& \lim _{E \uparrow 0} \int_{\mathbb{R}^{2}} \int_{\mathbb{R}^{2}}\left|F_{E}(x, y)\right|^{2} d x d y \\
& \quad=\int_{\mathbb{R}^{2}} \int_{\mathbb{R}^{2}}|V(x)||V(y)|\left|-\frac{1}{2 \pi} \ln \right| x-y\left|+\frac{\ln 2-\gamma}{2 \pi}\right|^{2} d x d y .
\end{aligned}
$$

Proof. Taking into account the definition of $F_{E}$, it suffices to prove the following three statements:

$$
\begin{gathered}
\lim _{E \uparrow 0} \int_{\mathbb{R}^{2}} \int_{\mathbb{R}^{2}}|V(x)||V(y)|\left|\ln (\sqrt{-E})\left(f(\sqrt{-E}|x-y|)+\frac{1}{2 \pi}\right)\right|^{2} d x d y=0 \\
\lim _{E \uparrow 0} \int_{\mathbb{R}^{2}} \int_{\mathbb{R}^{2}}|V(x)||V(y)||\ln | x-y\left|\left(f(\sqrt{-E}|x-y|)+\frac{1}{2 \pi}\right)\right|^{2} d x d y=0 \\
\lim _{E \uparrow 0} \int_{\mathbb{R}^{2}} \int_{\mathbb{R}^{2}}|V(x)||V(y)|\left|g(\sqrt{-E}|x-y|)-\frac{\ln 2-\gamma}{2 \pi}\right|^{2} d x d y=0 .
\end{gathered}
$$

Since $V$ is compactly supported and $f$ and $g$ are continuous with $f(0)=-\frac{1}{2 \pi}$ and $g(0)=\frac{\ln 2-\gamma}{2 \pi}$, one gets (2.10) and (2.11). As for (2.9) one can write

$$
\begin{aligned}
\ln (\sqrt{-E}) & \left(f(\sqrt{-E}|x-y|)+\frac{1}{2 \pi}\right) \\
& =\ln (\sqrt{-E}) \sqrt{-E}|x-y| \frac{f(\sqrt{-E}|x-y|)+\frac{1}{2 \pi}}{\sqrt{-E}|x-y|} .
\end{aligned}
$$

Let

$$
k(z)=\frac{f(z)+\frac{1}{2 \pi}}{z} .
$$

Since $f \in C^{\infty}\left(\mathbb{R}^{2}\right)$, it follows that $k$ is continuous (and, in particular, bounded on compact sets). Therefore (since $|x| \leq R$ and $|y| \leq R$ imply $|x-y| \leq 2 R$ ), there exists an $M>0$ such that

$$
k(\sqrt{-E}|x-y|) \chi_{\operatorname{supp}(V)}(x) \chi_{\operatorname{supp}(V)}(y) \leq M
$$

for any $E \in(-1,0)$ and any $x, y \in \mathbb{R}^{2}$. 
Since

$$
\lim _{E \uparrow 0} \ln (\sqrt{-E}) \sqrt{-E}=0,
$$

one gets (2.9).

Using (2.7) and the previous lemma, one immediately gets

$$
N(V) \leq 1+\int_{\mathbb{R}^{2}} \int_{\mathbb{R}^{2}}|V(x)||V(y)|\left|-\frac{1}{2 \pi} \ln \right| x-y\left|+\frac{\ln 2-\gamma}{2 \pi}\right|^{2} d x d y .
$$

The proof of Lemma 2.2 also shows that for $V \in L^{\infty}\left(\mathbb{R}^{2}\right)$ with $\operatorname{supp}(V)$ compact, we have

$$
\int_{\mathbb{R}^{2}} \int_{\mathbb{R}^{2}}|V(x)||V(y)|-\frac{1}{2 \pi} \ln |x-y|+\left.\frac{\ln 2-\gamma}{2 \pi}\right|^{2} d x d y<\infty
$$

\section{Estimate For a LARGER Class of POTENTIALS}

The result of Theorem 2.1 can be extended to any potential $V$ for which (2.12) holds. In order to prove this, the following lemma will be useful.

Lemma 3.1. Let $V$ be a real-valued measurable function such that

$$
\int_{\mathbb{R}^{2}} \int_{\mathbb{R}^{2}}|V(x)||V(y)|\left|C_{1} \ln \right| x-y\left|+C_{2}\right|^{2} d x d y<\infty .
$$

Then $V \in L^{1}\left(\mathbb{R}^{2}\right)$.

Proof. Let $V$ be a nonzero measurable function. Then there exists $a>0$ such that for $M=\left\{x \in \mathbb{R}^{2},|V(x)| \geq a\right\}$ one has $\lambda_{2}(M)>0$ ( $\lambda_{2}$ is the Lebesgue measure in $\left.\mathbb{R}^{2}\right)$. Furthermore, there exists a bounded open set $N \subset \mathbb{R}^{2}$ such that $\lambda_{2}(M \cap N)=b>0$.

Let $\epsilon>0$ be small enough such that

$$
\lambda_{2}\left(\left\{z \in \mathbb{R}^{2},|z| \in\left[e^{-C_{2} / C_{1}}-\epsilon, e^{-C_{2} / C_{1}}+\epsilon\right]\right\}\right) \leq \frac{b}{2} .
$$

For any $x \in \mathbb{R}^{2}$, let $N_{x}=\left\{y \in \mathbb{R}^{2},|y-x| \in\left[e^{-C_{2} / C_{1}}-\epsilon, e^{-C_{2} / C_{1}}+\epsilon\right]\right\}$. From (3.1) it follows that $\lambda_{2}\left(N_{x}\right) \leq \frac{b}{2}$ for any $x \in \mathbb{R}^{2}$.

Since $C_{1} \ln |z|+C_{2}=0$ if and only if $|z|=e^{-C_{2} / C_{1}}$, it follows that there exists a $c>0$ such that for a fixed $x \in \mathbb{R}^{2}$, one has

$$
\left|C_{1} \ln \right| x-y\left|+C_{2}\right|>c \text { for any } y \notin N_{x} .
$$

Now, for any $x \in \mathbb{R}^{2}$, since $\lambda_{2}(M \cap N)=b>0$ and $\lambda_{2}\left(N_{x}\right) \leq \frac{b}{2}$ one gets $\lambda_{2}\left(P_{x}\right)>\frac{b}{2}$, where $P_{x}=(M \cap N) \backslash N_{x}$. So

$$
\begin{aligned}
\int_{\mathbb{R}^{2}}|V(y)|\left|C_{1} \ln \right| x-y\left|+C_{2}\right|^{2} d x d y & \geq \int_{P_{x}}|V(y)|\left|C_{1} \ln \right| x-y\left|+C_{2}\right|^{2} d x d y \\
& \geq a \cdot c^{2} \cdot \lambda_{2}\left(P_{x}\right) \geq \frac{a c^{2} b}{2}>0 .
\end{aligned}
$$

Let $d=a c^{2} b / 2$. Since

$$
\int_{\mathbb{R}^{2}} \int_{\mathbb{R}^{2}}|V(x)||V(y)|\left|C_{1} \ln \right| x-y\left|+C_{2}\right|^{2} d x d y
$$




$$
=\int_{\mathbb{R}^{2}}|V(x)|\left(\int_{\mathbb{R}^{2}}|V(y)|\left|C_{1} \ln \right| x-y\left|+C_{2}\right|^{2} d y\right) d x \geq d\|V\|_{1},
$$

one gets $\|V\|_{1}<\infty$; so $V \in L^{1}\left(\mathbb{R}^{2}\right)$.

Proposition 3.2. Let $V$ be a real-valued measurable function such that

$$
\int_{\mathbb{R}^{2}} \int_{\mathbb{R}^{2}}|V(x)||V(y)|\left|C_{1} \ln \right| x-y\left|+C_{2}\right|^{2} d x d y<\infty .
$$

Then $V$ is a relatively form compact perturbation of $-\Delta$ that defines $-\Delta+V$ with $\sigma_{\text {ess }}(-\Delta+V)=[0, \infty)$.

Proof. $C_{1} \neq 0$ implies that $|V(x)||V(y)||\ln | x-y|+D|^{2} \in L^{1}\left(\mathbb{R}^{2} \times \mathbb{R}^{2}\right)$ where $D=C_{2} / C_{1}$. Since $V \in L^{1}\left(\mathbb{R}^{2}\right)$, one gets

$$
\begin{aligned}
& \int_{\mathbb{R}^{2}} \int_{\mathbb{R}^{2}}|V(x)||V(y)||\ln | x-\left.y\right|^{2} d x d y \\
& \quad \leq 2 \int_{\mathbb{R}^{2}} \int_{\mathbb{R}^{2}}|V(x)||V(y)||\ln | x-y|+D|^{2} d x d y+2 D^{2}\|V\|_{1}^{2}<\infty .
\end{aligned}
$$

From previous considerations, the integral kernel of $(-\Delta+1)^{-1}$ is

$$
\frac{1}{2 \pi} K_{0}(|x-y|)=(\ln |x-y|) f(|x-y|)+g(|x-y|),
$$

and therefore the integral kernel of $|V|^{1 / 2}(-\Delta+1)^{-1}|V|^{1 / 2}$ is

$$
K(x, y)=|V(x)|^{1 / 2}|V(y)|^{1 / 2}[(\ln |x-y|) f(|x-y|)+g(|x-y|)] .
$$

Since $f$ and $g$ are bounded, (3.4) implies that $K \in L^{2}\left(\mathbb{R}^{2} \times \mathbb{R}^{2}\right)$. Therefore, $|V|^{1 / 2}(-\Delta+1)^{-1}|V|^{1 / 2}$ is Hilbert-Schmidt. One can now write $(-\Delta+1)^{-1}=$ $(-\Delta+1)^{-1 / 2}(-\Delta+1)^{-1 / 2}$ and, using trace class ideals methods (see [10]), it follows that $(-\Delta+1)^{-1 / 2}|V|(-\Delta+1)^{-1 / 2}$ is Hilbert-Schmidt. This implies (cf. [8]) that $V$ is a relatively form compact perturbation of $-\Delta$ and therefore $V$ has relative form bound zero and $\sigma_{\text {ess }}(-\Delta+V)=\sigma_{\text {ess }}(-\Delta)=[0, \infty)$.

Theorem 3.3. Let $V$ be a real-valued measurable function such that

$$
B(V)=\int_{\mathbb{R}^{2}} \int_{\mathbb{R}^{2}}|V(x)||V(y)|\left|-\frac{1}{2 \pi} \ln \right| x-y\left|+\frac{\ln 2-\gamma}{2 \pi}\right|^{2} d x d y<\infty .
$$

Then

$$
N(V) \leq 1+\int_{\mathbb{R}^{2}} \int_{\mathbb{R}^{2}}|V(x)||V(y)|\left|-\frac{1}{2 \pi} \ln \right| x-y\left|+\frac{\ln 2-\gamma}{2 \pi}\right|^{2} d x d y .
$$

Proof. As was shown before, one can assume, without loss of generality, that $V \leq 0$. For any positive integer $N$, let

$$
V_{N}(x)=\max (V(x),-N) \chi_{(-N, N)}(x) .
$$

Obviously, $V_{N} \in L^{\infty}$ and $\operatorname{supp}\left(V_{N}\right) \subset[-N, N]$ for any $N$. So from Theorem 2.1 one gets

$$
N\left(V_{N}\right) \leq 1+\int_{\mathbb{R}^{2}} \int_{\mathbb{R}^{2}}|V(x)||V(y)|\left|-\frac{1}{2 \pi} \ln \right| x-y\left|+\frac{\ln 2-\gamma}{2 \pi}\right|^{2} d x d y .
$$

Since $\left(-\Delta+V_{N}\right) \varphi \rightarrow(-\Delta+V) \varphi$ for any $\varphi$ in the domain of $(-\Delta)^{1 / 2}$ it follows, using a result from [6], that $\left(-\Delta+V_{N}\right) \rightarrow(-\Delta+V)$ in strong resolvent sense, 
which implies that $N(V) \leq \lim \sup _{N \rightarrow \infty} N\left(V_{N}\right)$. Since $\left|V_{N}\right| \uparrow|V|$ one gets, using the monotone convergence theorem, that

$$
N(V) \leq 1+\int_{\mathbb{R}^{2}} \int_{\mathbb{R}^{2}}|V(x)||V(y)|\left|-\frac{1}{2 \pi} \ln \right| x-y\left|+\frac{\ln 2-\gamma}{2 \pi}\right|^{2} d x d y .
$$

Proposition 3.4. Let $V \in L^{1}\left(\mathbb{R}^{2}\right)$ be such that $V[\ln (1+|x|)]^{2} \in L^{1}\left(\mathbb{R}^{2}\right)$ and $|V|^{*}[\ln |x|]^{2} \chi_{\{|x| \leq 1\}} \in L^{1}\left(\mathbb{R}^{2}\right)$ where $|V|^{*}$ is the symmetric decreasing rearrangement of $|V|$. Then the integral in Theorem 3.3 is finite (i.e., $B(V)<\infty$ ) and

$$
\begin{aligned}
N(V) \leq 1 & +C_{3}\|V\|_{1}^{2}+C_{4}\|V\|_{1} \int_{\mathbb{R}^{2}}|V(x)|[\ln (1+|x|)]^{2} d x \\
& +C_{5}\|V\|_{1} \int_{\mathbb{R}^{2}}|V|^{*}(x)[\ln |x|]^{2} \chi_{\{|x| \leq 1\}} d x
\end{aligned}
$$

where $C_{3}, C_{4}$ and $C_{5}$ are positive constants.

Proof. As in (3.4),

$$
\begin{aligned}
B(V)= & \int_{\mathbb{R}^{2}} \int_{\mathbb{R}^{2}}|V(x)||V(y)|\left|C_{1} \ln \right| x-y\left|+C_{2}\right|^{2} d x d y \\
& \leq 2 C_{1}^{2} \int_{\mathbb{R}^{2}} \int_{\mathbb{R}^{2}}|V(x)||V(y)||\ln | x-\left.y\right|^{2} d x d y+2 C_{2}^{2}\|V\|_{1}^{2} .
\end{aligned}
$$

Let $S_{1}=\left\{(x, y) \in \mathbb{R}^{2} \times \mathbb{R}^{2},|x-y| \geq 1\right\}$ and $S_{2}=\left(\mathbb{R}^{2} \times \mathbb{R}^{2}\right) \backslash S_{1}$. Then, since $|x-y| \leq(1+|x|)(1+|y|)$, one gets that

$$
|\ln | x-\left.y\right|^{2} \leq 2[\ln (1+|x|)]^{2}+2[\ln (1+|y|)]^{2}
$$

for any $(x, y) \in S_{1}$ and therefore

$$
\int_{S_{1}}|V(x)||V(y)||\ln | x-\left.y\right|^{2} d x d y \leq 4\|V\|_{1} \int_{\mathbb{R}^{2}}|V(x)|[\ln (1+|x|)]^{2} d x .
$$

Now let $h(z)=\left.|\ln | z\right|^{2} \chi_{\{|z| \leq 1\}}$. Using the Brascamp-Lieb-Luttinger inequality [4] one gets:

$$
\begin{aligned}
\int_{S_{2}}|V(x)||V(y)||\ln | x-\left.y\right|^{2} d x d y & =\int_{\mathbb{R}^{2}} \int_{\mathbb{R}^{2}}|V(x)||V(y)| h(x-y) d x d y \\
& \leq \int_{\mathbb{R}^{2}} \int_{\mathbb{R}^{2}}|V|^{*}(x)|V|^{*}(y) h^{*}(x-y) d x d y \\
& =\int_{\mathbb{R}^{2}} \int_{\mathbb{R}^{2}}|V|^{*}(x)|V|^{*}(y) h(x-y) d x d y
\end{aligned}
$$

Since for any $y \in \mathbb{R}^{2}$,

$$
\int_{\{|x-y| \leq 1\}}|V|^{*}(x)|\ln | x-\left.y\right|^{2} d x \leq \int_{\{|x| \leq 1\}}|V|^{*}(x)(\ln |x|)^{2} d x,
$$

one gets, using (3.8),

$$
\int_{S_{2}}|V(x)||V(y)||\ln | x-\left.y\right|^{2} d x d y \leq\|V\|_{1} \int_{\mathbb{R}^{2}}|V|^{*}(x)[\ln |x|]^{2} \chi_{\{|x| \leq 1\}} d x .
$$

Combining (3.6), (3.7) and (3.9) one gets (3.5) with $C_{3}=2 C_{2}^{2}=(\ln 2-\gamma)^{2} / 2 \pi^{2}$, $C_{4}=8 C_{1}^{2}=2 / \pi^{2}$ and $C_{5}=2 C_{1}^{2}=1 / 2 \pi^{2}$. 
Remarks. 1. Since $N(V) \leq N\left(-V_{-}\right)$, one can improve the estimates in Theorem 3.3 and Proposition 3.4 by replacing (on the right-hand side) $V$ with $V_{-}$.

2. As mentioned before, for large coupling constants, $B(\lambda V), \widetilde{B}(\lambda V) \sim \lambda^{2}$. Using the trace class ideals methods developed in [10], it should be possible to get bounds $\sim \lambda^{1+\epsilon}$, for any $\epsilon>0$. However, it is not clear how to get a bound $\sim \lambda$ as conjectured by Khuri, Martin and $\mathrm{Wu}$ in [5].

\section{ACKNOWLEDGEMENTS}

I would like to thank B. Simon for proposing the problem and both R. Killip and B. Simon for useful discussions.

Note added in proof. After the submission of this paper I learned of further related results: N. Setô [15], R. Newton [14] and M. Solomyak [17]. Readers interested in the one-dimensional problem should refer to B. Simon [16] and M. Klaus [13]. I would like to thank P. Exner for bringing some of these papers to my attention.

\section{REFERENCES}

[1] M. Abramowitz and I. Stegun (editors), Handbook of Mathematical Functions, with Formulas, Graphs, and Mathematical Tables, Dover Publications, New York, 1972. MR 94b:00012

[2] G. B. Arfken and H. J. Weber, Mathematical Methods for Physicists, Academic Press, San Diego, CA, 1995. MR 98a:00001

[3] M. S. Birman, On the spectrum of singular boundary-value problems, Mat. Sb. (N.S.) 55 (97) (1961) 125-174, English translation, Amer. Math. Soc. Transl. 53 (1966), 23-80. MR 26:463

[4] H. J. Brascamp, Elliott H. Lieb and J. M. Luttinger, A general rearrangement inequality for multiple integrals, J. Funct. Anal. 17 (1974), 227-237. MR 49:10835

[5] N. N. Khuri, A. Martin, and T. T. Wu, Bound states in $n$ dimensions (especially $n=1$ and $n=2)$, Few Body Systems 31 (2002), 83-89.

[6] M. Reed and B. Simon, Methods of Modern Mathematical Physics, I: Functional Analysis, Academic Press, New York, 1972. MR 58:12429a

[7] M. Reed and B. Simon, Methods of Modern Mathematical Physics, II: Fourier Analysis, Self Adjointness, Academic Press, New York, 1975. MR 58:12429b

[8] M. Reed and B. Simon, Methods of Modern Mathematical Physics, IV: Analysis of Operators, Academic Press, New York, 1978. MR 58:12429c

[9] J. Schwinger, On the bound states of a given potential, Proc. Nat. Acad. Sci. U.S.A. 47 (1961), 122-129. MR 23:B2833

[10] B. Simon, Trace Ideals and Their Applications, London Math. Soc. Lecture Note Series, Vol. 35, Cambridge University Press, Cambridge, 1979. MR 80k:47048

[11] B. Simon, The bound state of weakly coupled Schrödinger operators in one and two dimensions, Ann. Phys. 97 (1976), 279-288. MR 53:8646

[12] B. Simon, On the number of bound states of two-body Schrödinger operators: A review, in Studies in Mathematical Physics, Essays in Honor of Valentine Bargmann, Princeton University Press, Princeton, 1976, pp. 305-326.

[13] M. Klaus, On the bound state of Schrödinger operators in one dimension, Ann. Physics 108 (1977), no. 2, 288-300. MR 58:20010

[14] R. G. Newton, Bounds on the number of bound states for the Schrödinger equation in one and two dimensions, J. Operator Theory 10 (1983), no. 1, 119-125. MR 85e:81029

[15] N. Setô, Bargmann's inequalities in spaces of arbitrary dimension, Publ. Res. Inst. Math. Sci. 9 (1973/74), 429-461. MR 49:5596

[16] B. Simon, An introduction to the self-adjointness and spectral analysis of Schrödinger operators in The Schrödinger Equation (W. Thirring and P. Urban, eds.), Acta Phys. Aus. Suppl. 17, Springer, Vienna, 1977, pp. 19-42. 
[17] M. Solomyak, Piecewise-polynomial approximation of functions from $H^{l}\left((0,1)^{d}\right), 2 l=d$, and applications to the spectral theory of the Schrödinger operator, Israel J. Math. 86 (1994), no. 1-3, 253-275. MR 95e:35151

Department of Mathematics 253-37, California Institute of Technology, Pasadena, CALIFornia 91125

E-mail address: mihai@its.caltech.edu 